\title{
Regulatory roles of interferon-inducible protein 204 on differentiation and vasculogenic activity of endothelial progenitor cells
}

\author{
Junjie Yang ${ }^{1,5^{\dagger}}$, Xiaofei Zhang ${ }^{1 \dagger}$, Zhenao Zhao ${ }^{1+}$, Xizhe $\mathrm{Li}^{2}, \mathrm{Xu} \mathrm{Wang}^{1}$, Ming Chen ${ }^{1}$, Bo Song ${ }^{3}$, Masaaki $\mathrm{li}^{4^{*}}$
} and Zhenya Shen ${ }^{1 *}$

\begin{abstract}
Background: Endothelial progenitor cells (EPCS) have shown great potential in angiogenesis either by their differentiation into endothelial cells or by secretion of angiogenic factors. Interferon-inducible protein 204 (Ifi204) has been reported to participate in the regulation of cell growth and differentiation. However, its role in differentiation of EPCs remains unknown. We proposed that Ifi204 could modulate the differentiation and regenerative abilities of EPCs.

Methods: Ifi204-expressing lentivirus and Ifi204 siRNA were introduced into EPCs to overexpress and suppress the expression of Ifi204. Using fluorescence-activated cell sorting, immunocytochemistry, and quantitative PCR, endothelial markers including CD31, VE-cadherin, and VWF were detected in the modified EPCs. An in-vitro incorporation assay and a colony-forming assay were also performed.

Results: Evidence showed that Ifi204 inhibition decreased the endothelial differentiation and vasculogenic activities of EPCs in vitro. In mice with hindlimb ischemia, downregulation of Ifi204 in EPCs, which was tracked by our newly synthesized nanofluorogen, impaired neovascularization, with a corresponding reduction in hindlimb blood reperfusion by postoperative day 14 .
\end{abstract}

Conclusions: Ifi204 is required for EPC differentiation and neovascularization in vitro and in vivo. The regulatory roles of Ifi204 in EPC differentiation may benefit the clinical therapy of ischemic vascular diseases.

Keywords: Endothelial progenitor cells, Ifi204, Endothelial differentiation, Vasculogenesis, Hindlimb ischemia

\section{Background}

In 1997, Asahara et al. [1] revolutionized the traditional concept of postnatal neovascularization, proposing that immature endothelial progenitor cells (EPCs) circulate in the adult blood and participate in postnatal angiogenesis. This idea was met with great enthusiasm because it suggested that EPCs could be derived from peripheral blood and used as a novel therapy in patients with vascular

\footnotetext{
*Correspondence: masaii@osaka-med.ac.jp; uuzyshen@aliyun.com

${ }^{\dagger}$ Equal contributors

${ }^{4}$ Division of Research Animal Laboratory and Translational Medicine, Research and Development Center, Osaka Medical College, 2-7 Daigaku-machi,

Takatsuki, Osaka 569-8686, Japan

'Institute for Cardiovascular Science \& Department of Cardiovascular Surgery of The First Affiliated Hospital, Soochow University, 188 Shizi Street, Suzhou 215006, China Full list of author information is available at the end of the article
}

disease, and thus was followed by booming research on EPCs over the last decades. Upon ischemic stimuli, EPCs are recruited to ischemic tissue and enhance angiogenesis either by incorporation into neovascularization [2-5] or by secreting angiogenic and cytoprotective cytokines and growth factors [6-10]. To optimize therapeutic outcomes, researchers have developed techniques such as genetic modification of EPCs and so on [11-14].

Interferon-inducible protein 204 (Ifi204), a member of the interferon-inducible p200 family of proteins, has participated in the regulation of cell growth and differentiation [15]. Evidence has shown that Ifi204 could promote muscle cell differentiation by sequestering regulatory factors that block the differentiation process $[16,17]$. Very recently, our group reported that Ifi204 is downregulated 
in the bone marrow cells of diabetes mellitus type 2 mice [18]. Because EPCs in diabetes mellitus have been reported to be impaired in regenerative potency, we thus proposed that Ifi204 could modulate the differentiation and regenerative abilities of EPCs. Through overexpression and inhibition of Ifi204 in EPCs, we examined the role of Ifi204 in EPC differentiation and vasculogenesis ability in vitro, and their ability for neovascularization in a hindlimb ischemia model.

\section{Methods}

\section{Animals}

All animal procedures were approved by the Ethic Committee of Soochow University and carried out in accordance with the Guidelines for the Care and Use of Research Animals established by Soochow University. C57/BL6 mice (8 weeks old, male) were housed at the Animal Facility of Soochow University on a 12-hour light/dark cycle with free access to water and standard mouse food.

\section{Isolation and identification of EPCs}

Bone marrow cells were isolated from mouse bones as described previously [19]. Briefly, cells were collected from all bones smashed with a mortar and a pestle, and were further isolated by density-gradient centrifugation with Histopaque 1083 (Sigma, St. Louis, MO, USA). Cells were cultured in Endothelial Cell Basal Medium-2 (Lonza, Basel, Switzerland), supplemented with EGM-2 MV SingleQuots (Lonza) and $10 \%$ fetal bovine serum. All the floating cells were washed out with fresh culture medium 2 days after cell plating, and were further cultured for 14 days before experiments. Then $5 \mu \mathrm{g} / \mathrm{ml}$ of DiI-Ac-LDL solution (Biomedical Technologies Inc., Stoughton, MA, USA) was added to the cells and culture continued for 4 hours. The cells were then fixed by $4 \%$ paraformaldehyde (PFA)/PBS and stained with DAPI (Beyotime Biotechnology, Shanghai, China). Endocytosis of Ac-LDL was observed under a fluorescent microscope (Olympus, Tokyo, Japan).

\section{Transfection of Ifi204 in EPCs}

Mouse Ifi204 siRNAs were synthesized by GenePharma Co. Ltd (Shanghai, China). The sequences for Ifi204 siRNAs were designed as follows: sense ( $5^{\prime}$ to $\left.3^{\prime}\right)$, CCAACAAUGG UUAUCUCAATT; and anti-sense ( $5^{\prime}$ to $\left.3^{\prime}\right)$, UUGAGA UAACCAUUGUUGGTT. Cell transfection was performed with Lipofectamine 2000 (Invitrogen, Carlsbad, CA, USA), according to the manufacturer's protocols. Briefly, the cells in $60 \mathrm{~mm}$ dishes were transfected with $200 \mathrm{pmol}$ of Ifi204 siRNA and the scramble siRNA separately. Forty-eight hours post transfection, the cells were harvested and the efficiency of transfection was confirmed using reverse transcription quantitative PCR (RT-qPCR). At the same time, the transfected cells were further used in the invitro assays.

\section{Synthesis of lentivirus overexpressing Ifi204 and transduction of lentivirus in EPCs}

Ifi204 full-length CDS was cloned into the NotI/BamHI sites of the LV5 vector (EF1A-GFP-PURO). Positive clones of LV5-Ifi204 vectors were identified by DNA sequencing. Recombinant LV5 lentivirus containing Ifi204 gene was produced in the HEK 293 packaging cell line. For infection, cells were exposed to viral supernatant at a multiplicity of infection (MOI) of 50 for 24 hours. The efficiency of transduction was estimated by calculating the percentage of green fluorescence protein (GFP)-positive cells and RT-qPCR.

\section{Fluorescence-activated cell sorting analysis}

To detect endothelial lineage antigens on EPCs, fluores cence-activated cell sorting (FACS) analysis was conducted 4 days after siRNA transfection or lentivirus transduction of EPCs. Surface expression of CD133 and VE-cadherin was determined by phycoerythrin (PE)-conjugated antibody (e-Bioscience, San Diego, CA, USA) against mouse CD133 and VE-cadherin. FITC-conjugated anti-mouse PECAM (CD31) antibody (e-Bioscience) was used to determine the surface expression of CD31. Isotypeidentical antibodies served as negative controls (BioLegend, San Diego, CA, USA). The staining was performed for 20 minutes at $4{ }^{\circ} \mathrm{C}$, followed by quantitative analysis with MoFlo XDP (Beckman Coulter, Brea, CA, USA).

\section{Immunocytochemistry}

Attached cells were briefly fixed with ice-cold acetone and blocked in antibody dilution buffer (2\% BSA/PBS) for 1 hour at room temperature. After removal of the blocking solution, primary antibodies anti-VE-cadherin antibody (1:100; Santa Cruz, Dallas, TX, USA) and antiCD31 antibody (1:100; Santa Cruz) were added and cells were incubated at $4{ }^{\circ} \mathrm{C}$ overnight. The cells were then washed and incubated with Alexa 488 donkey anti-goat IgG and Alexa 488 goat anti rabbit IgG (Invitrogen, Carlsbad, CA, USA) for 30 minutes at room temperature. Immunofluorescence was observed under a fluorescent microscope (Olympus).

\section{Real-time RT-PCR analysis}

Cellular mRNA was extracted from each sample using PureLink RNA Mini Kit (Ambion, Carlsbad, CA, USA) according to the manufacturer's instructions. cDNA was synthesized using PrimeScript RT reagent Kit (TAKARA, Tokyo, Japan). For qPCR, the converted cDNA samples $(2 \mu \mathrm{l})$ were amplified in a final volume of $10 \mu \mathrm{l}$ using SYBR Green Master Mix reagent (Applied Biosystems, Foster City, CA, USA) and gene-specific primers with the 
Table 1 Primer sequences for real-time quantitative PCR

\begin{tabular}{lll}
\hline Gene name & Forward primer & Reverse primer \\
\hline GAPDH & ACATCATCCCTGCATCCACT & CACATTGGGGGTAGGAACAC \\
CD31 & TGGCCAGTCACTTGAAGACA & CAGTTGTTGTAGCCAGCCATT \\
VE-cadherin & TACTCAGCCCTGCTCTGGT & GCTTGCAGAGGCTGTGTCTT \\
VWF & ACGCCATCTCCAGATTCAAG & AAGCATCTCCCACAGCATTC \\
VEGF & AGCACAGCAGATGTGAATGC & AATGCTTTCTCCGCTCTGAA
\end{tabular}

StepOne Plus real-time PCR system (Applied Biosystems). Sequences of the specific primers used in RT-PCR are presented in Table 1 . The mean cycle threshold values were used to calculate gene expression levels with normalization to mouse GAPDH. Quantitative analyses were based on three independent biological samples.

\section{In-vitro vasculogenesis assay}

Human umbilical cord-derived endothelial cells (HUVECs) and EPCs with Ifi204 siRNA transfection or LV5-Ifi204 infection were used for tube formation assays as described previously [19-21]. Fifty microliters of chilled Matrigel matrix (BD, Bedford, MA, USA) was added into each well of the 96-well plate and incubated at $37{ }^{\circ} \mathrm{C}$ for 30 minutes. EPCs were labeled with DiI (Invitrogen, Eugene, OR, USA) for 20 minutes. HUVECs together with DiI-labeled EPCs in $100 \mu \mathrm{l}$ of $10 \% \mathrm{FBS} / \mathrm{EGM}-2 \mathrm{MV}$ medium were added to each well. The number of incorporated Dil-labeled cells into tubes was counted under a fluorescent microscope (Olympus).

\section{EPC colony-formation assay}

EPCs with inhibition or overexpression of Ifi204 were cultured in methylcellulose-based medium (MethoCultM3236; Stem Cell Technologies, Vancouver, BC, Canada) supplemented with VEGF (50 ng/ml; PeproTech, Rocky Hill, NJ, USA), SCF (100 ng/ml; PeproTech), IL-3 (20 ng/ml; PeproTech), EGF (50 ng/ml; PeproTech), bFGF (50 ng/ml; PeproTech), IGF-1 (50 ng/ml; PeproTech), and 30 \% FBS (Sigma). EPC colonies were manually counted under a phase contrast microscope (Olympus) after culturing 1000 cells for 14 days. Endothelial phenotype of the EPC colonies was confirmed by uptake of DiI-Ac-LDL and FITC-conjugated isolectin B4 (Sigma Chemical Co., Milwaukee, WI, USA).
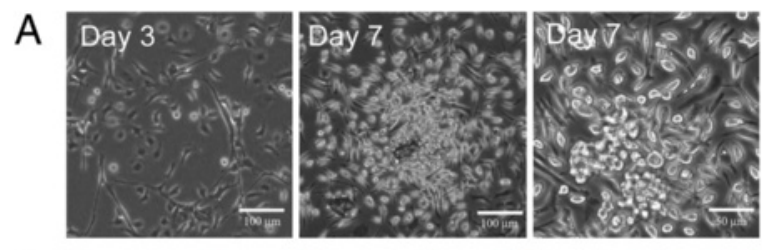

B
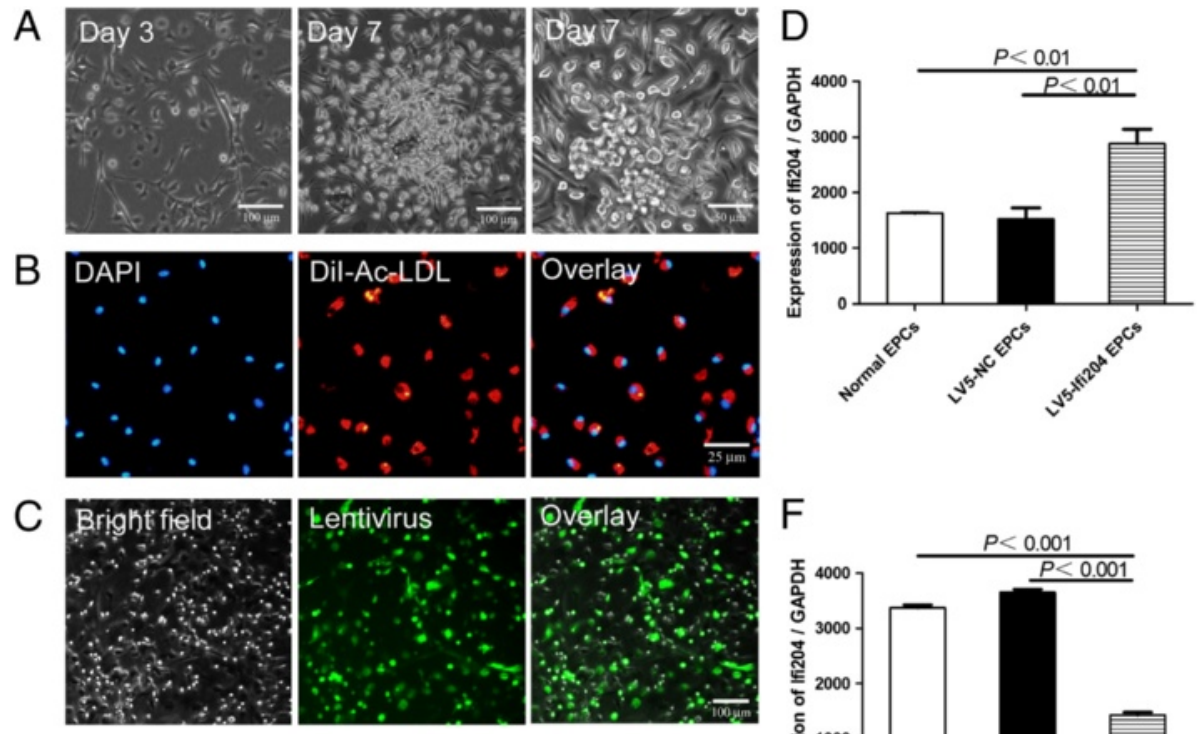

E
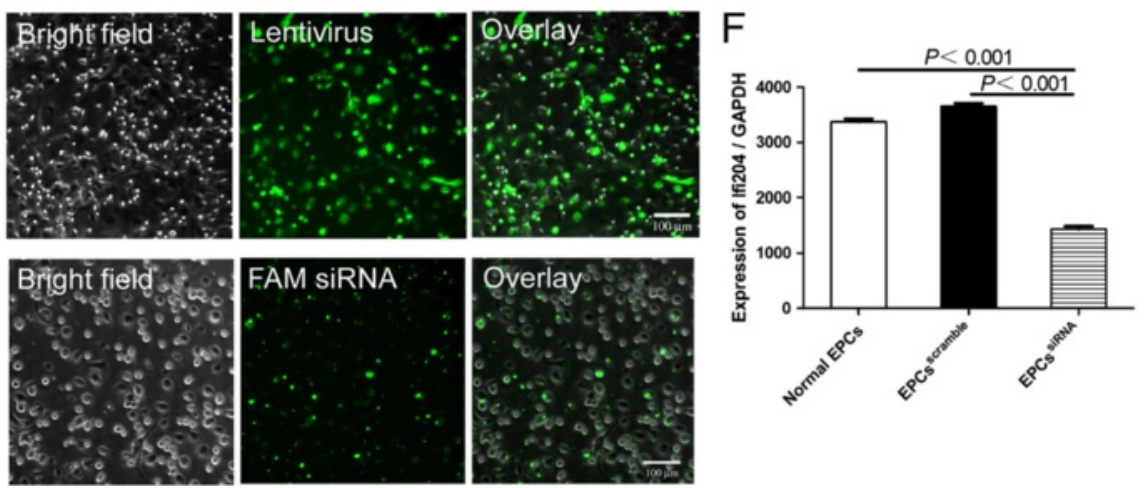

Fig. 1 Culture of EPCs and modulation of Ifi204 in EPCs. a Morphology of mouse bone marrow-derived EPCs cultured at day 3 and day 7. Bar, $100 \mu \mathrm{m}$ and $50 \mu \mathrm{m}$. b Uptake of Dil-Ac-LDL by EPCs. Bar, $25 \mu \mathrm{m}$. c Infection of Ifi204 lentivirus in EPCs. Bar, $50 \mu \mathrm{m}$. $\mathbf{d}$ Expression of Ifi204 in EPCs after transduction of Ifi204 expressing lentivirus $(n=3)$. $P<0.01$, LV5-Ifi204 EPCs vs LV-NC EPCs and normal EPCs. e Transfection of Ifi204 siRNA in EPCs. Bar, $100 \mu \mathrm{m}$. f Expression of Ifi204 in EPCs after transfection of Ifi204 siRNA $(n=3) . P<0.001$, EPCs ${ }^{\text {siRNA }}$ vs normal EPCs and EPCs $s^{\text {scramble }}$. EPC endothelial progenitor cell 
Hindlimb ischemia model and cell transplantation

Mice aged 8-10 weeks were anesthetized with $160 \mathrm{mg} / \mathrm{kg}$ pentobarbital i.p. The common femoral artery, including the superficial and other arterial branches as well as veins, were ligated and excised to generate the hindlimb ischemia model as described previously [22]. EPCs were administered intramuscularly immediately after induction of ischemia. For the study of EPC tracking, cells were incubated with our newly synthesized nanofluorogen [23], a bolaamphiphile with a tetraphenylethene (TPE) unit attached and two pyridinium salt terminated alkyl groups (TPE-11). Cells were washed with PBS and incubated with TPE-11 at a concentration of $16 \mu \mathrm{g} / \mathrm{ml}$ overnight. The cells were then collected and 50,000 cells were injected into the gastrocnemius muscle (two sites) and lower ligation sites (two sites) per mouse. Laser Doppler perfusion imaging (LDPI; Perimed Instruments AB, Stockholm, Sweden) was conducted to measure blood flow recovery ratio (ischemia/nonischemia). The perfusions of the ischemic and nonischemic limbs were calculated on the basis of colored histogram pixels, and expressed as the ratio of ischemic to nonischemic hindlimb perfusion.

\section{Detection of neovascularization in the hindlimb ischemia model}

To visualize functional vessels with blood flow, Griffonia (Bandeiraea) Simplicifolia Lectin I (BSL I; Vector Laboratories, Burlingame, CA, USA) was infused into mouse heart 15 minutes before sacrifice. Ischemic muscle samples were embedded in OCT compound (Sakura, Tokyo, Japan), snap-frozen in liquid nitrogen, and cut at a thickness of $6 \mu \mathrm{m}$ with a Leica CM 1950 Cryomicrotome (Carl Zeiss AG, Jena, Germany). Frozen sections were incubated with anti-BSL I antibody (Vector Laboratories) overnight at $4{ }^{\circ} \mathrm{C}$, followed by staining with Alexa fluor 594-conjugated anti-rabbit antibody (Invitrogen) to identify capillaries in the ischemic tissue. Images were examined with a fluorescent microscope (Olympus). Cell incorporation into neovascularization was detected by double-positivity of BSL I and TPE-11.
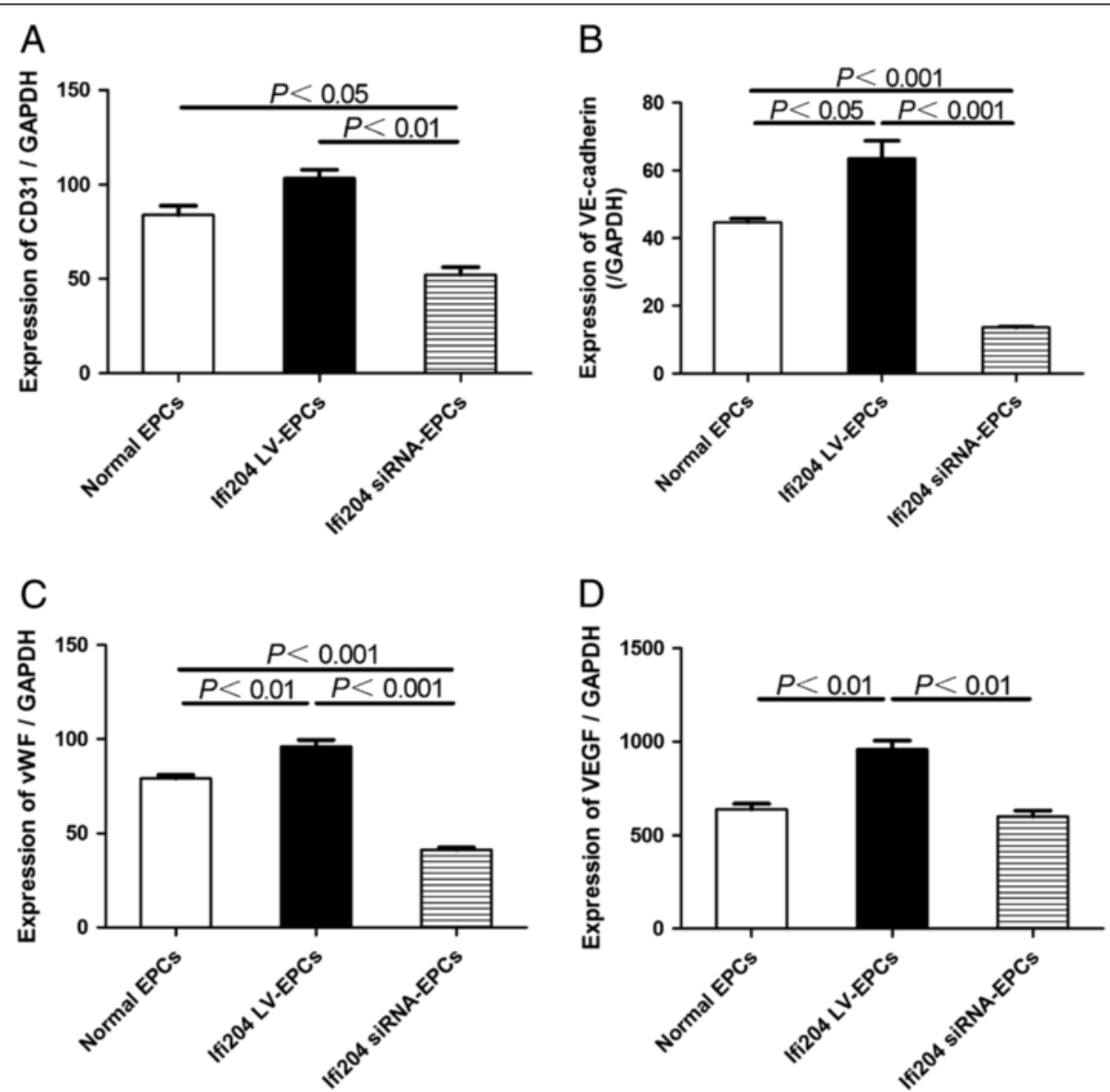

Fig. 2 qPCR analysis of endothelial markers and growth factors in different EPC groups. mRNA expression levels of CD31 a, VE-cadherin $\mathbf{b}$, vWF c, and VEGF $\mathbf{d}$ in 14-day cultured cells were assessed by quantitative real-time RT-PCR. The mRNA expressions were normalized to GAPDH ( $n=3$ ). All assays were performed in triplicate and demonstrated similar results. EPC endothelial progenitor cell 
Statistical analysis

Comparison of multiple groups was performed by ANOVA. Two-group analysis was performed by Student $t$ test. Values were expressed as means \pm SEM. $P<0.05$ was considered significant. Continuous variables are expressed as mean \pm SD or SEM.

\section{Results}

EPC culture and regulation of Ifi204 expression in EPCs In the culture medium of $10 \%$ FBS/EGM-2MV, adherent cells changed their morphology to spindle shape after 3 days. Seven days later, most of the cells formed colonies as reported previously (Fig. 1a). As a characteristic of
EPCs, cells could uptake Dil-Ac-LDL (Fig. 1b). We constructed a lentivirus vector with Ifi204 full-length CDS cloned into the NotI/BamHI sites. EPCs were infected with LV5-Ifi204 for 24 hours (Fig. 1c). The expression of Ifi204 in EPCs after lentivirus transduction reached about twofold compared with that of normal EPCs (Fig. 1d). We also transfected Ifi204 siRNA into EPCs (Fig. 1e), which inhibited the expression of Ifi204 by $58 \pm 0.03 \%$ (Fig. 1f).

\section{Downregulation of Ifi204 inhibited EPC differentiation} We next determined the expression profiles of endothelial cell-lineage markers in modified EPCs in comparison

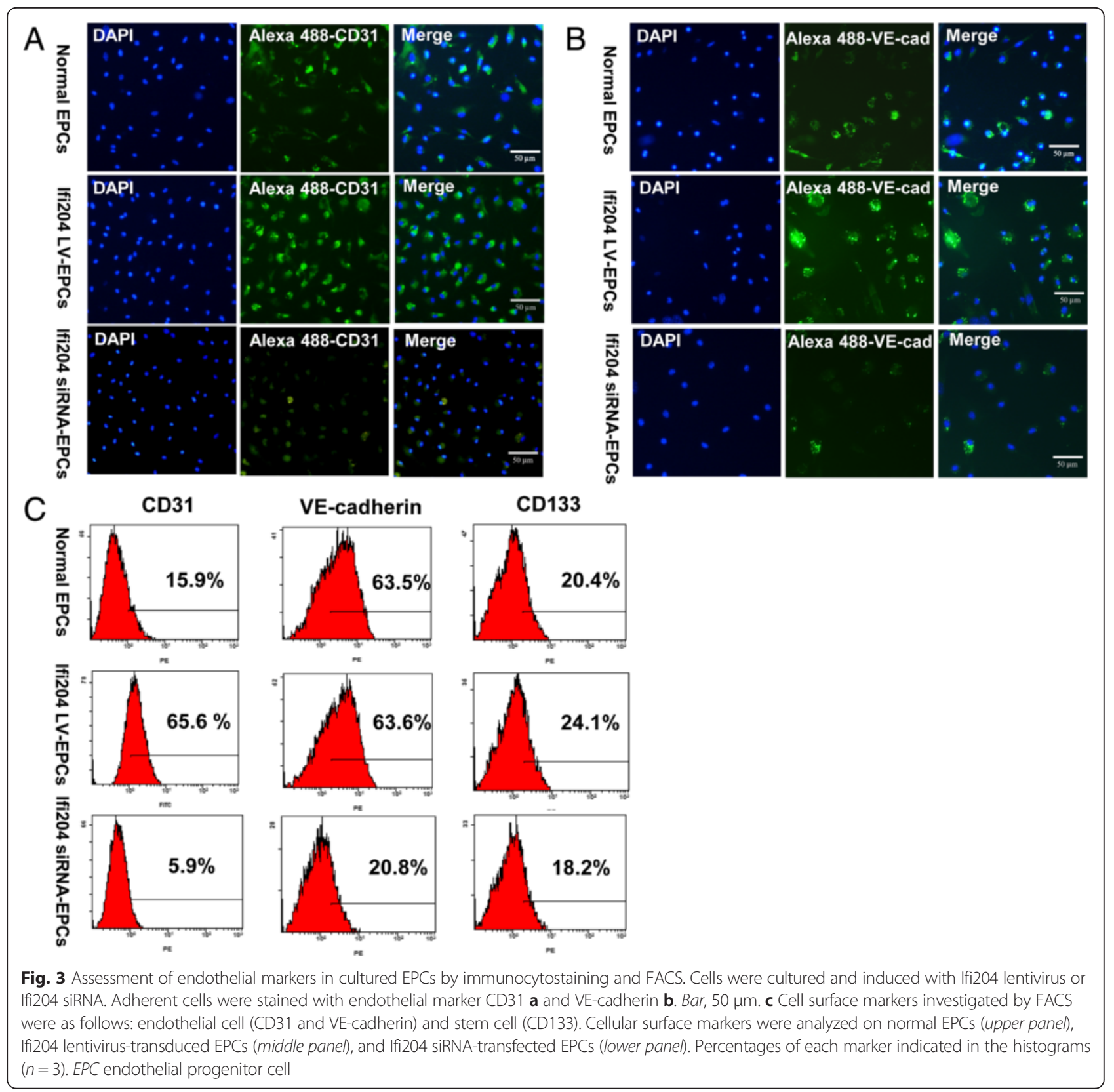


with normal EPCs. As shown in Fig. 2, the mRNA expression levels of CD31, VE-cadherin, vWF, and VEGF in Ifi204 LV-transduced EPCs were at least twofold those in Ifi204 siRNA-transfected EPCs (CD31 and VEGF in Ifi204 LV-EPCs vs Ifi204 siRNA-EPCs, $P<0.01$; VEcadherin and vWF in Ifi204 LV-EPCs vs Ifi204 siRNAEPCs, $P<0.001$ ), and also higher than those in normal EPCs (vWF and VEGF in Ifi204 LV-EPCs vs normal EPCs, $P<0.01$; VE-cadherin in Ifi204 LV-EPCs vs normal EPCs, $P<0.05)$. Most of the Ifi204 LV-transduced EPCs expressed CD31 (Fig. 3a) and VE-cadherin (Fig. 3b), but they were dramatically decreased in Ifi204 siRNA-transfected EPCs. We also analyzed the expression of endothelial celllineage markers on EPCs by FACS (Fig. 3c). EPCs infected with Ifi204 lentivirus had higher expression of CD31, VE-cadherin, and CD133 than normal EPCs. In contrast, inhibition of Ifi204 reduced the expression of these cell surface markers in EPCs. These results demonstrated a differentiation inhibitory effect of Ifi204 knockdown in EPCs.
In-vitro vasculogenic activity of EPCs was modulated by the expression of Ifi204

To determine whether Ifi204 could modulate the vasculogenic activity of EPCs, we carried out a tube-forming assay on Matrigel using HUVECs and EPCs as described previously [19-21]. As shown in Fig. 4, overexpression of Ifi204 in EPCs significantly enhanced the numbers of incorporated cells into tube structures of HUVECs $(P<0.001$, Ifi204 LV-EPCs: $43 \pm 4$ cells/HPF vs normal EPCs: $31 \pm 2$ cells/HPF and Ifi204 siRNA-EPCs: $19 \pm 3$ cells/HPF). Inhibition of Ifi204 in EPCs, however, prohibited the incorporation of EPCs into tube structures of HUVECs. The in-vitro angiotube assay revealed that the expression level of Ifi204 in EPCs was closely correlated with the angiogenesis abilities of the cells.

\section{Colony-forming ability of EPCs was slightly enhanced by Ifi204 siRNA transfection}

Subsequently, we explored the influence of Ifi204 on colony-formation ability, representing functional stem
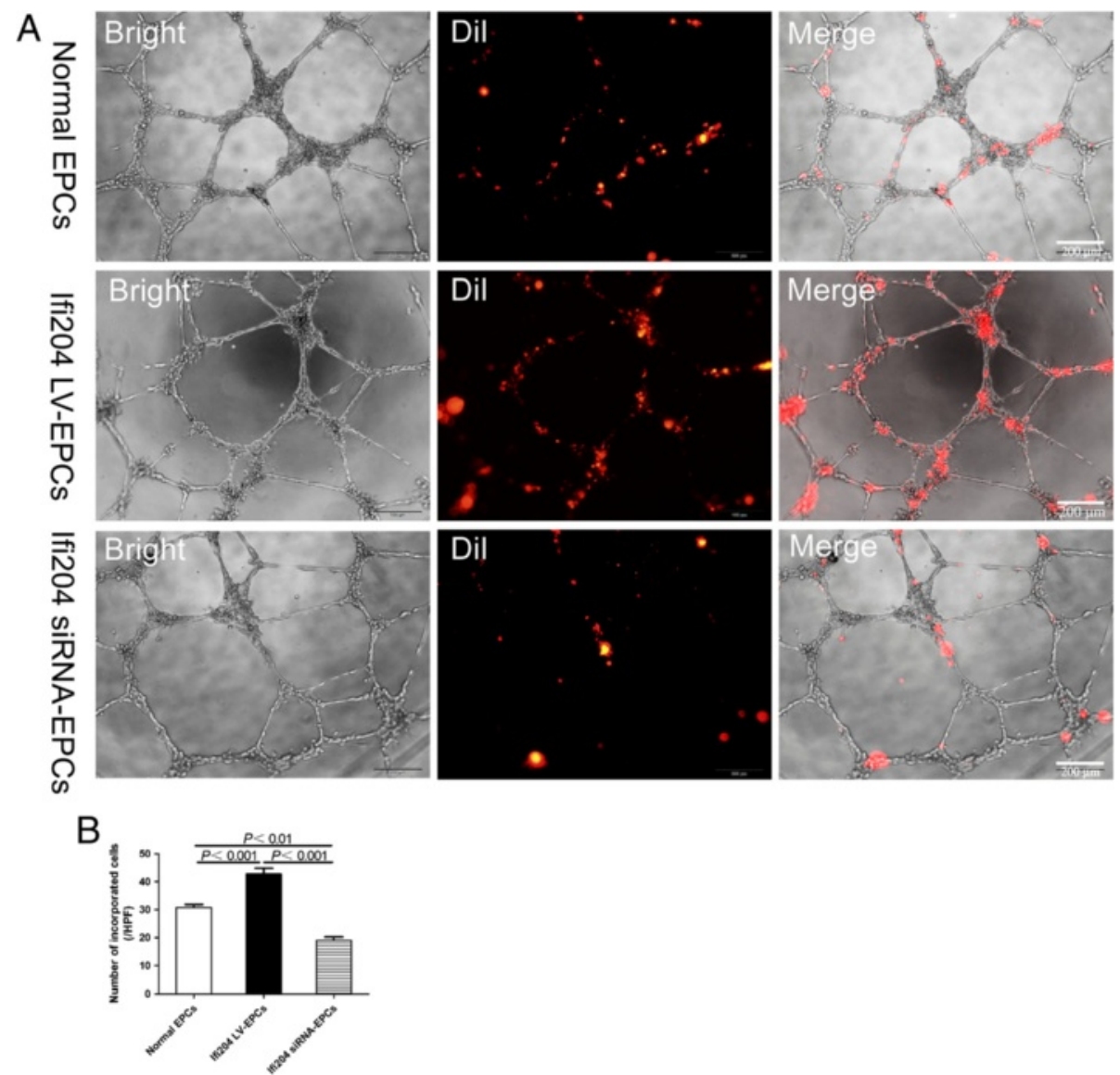

Fig. 4 Tube formation assay by HUVECs incorporated with modified EPCS. Dil-labeled cells and HUVECs were seeded onto Matrigel-coated 96-well plates in $10 \%$ FBS/EBM2-MV without growth factors. After 24 hours in culture, incorporation of each cell population into tube-like structures formed with HUVECs was evaluated under fluorescence microscopy. a Morphology of cultured HUVECs forming a network on Matrigel with the incorporation of Dil-positive cells. Bar, $200 \mu \mathrm{m}$. b Number of incorporated Dil-positive cells into tubular structures was counted and averaged. All assays demonstrated similar results $(n=4)$. EPC endothelial progenitor cell 
cell parameters. Colonies formed by EPCs could uptake FITC isolectin B4 and Dil-Ac-LDL (Fig. 5a). In EPCs, overexpression of Ifi204 increased the numbers of colonies by about twofold compared with downexpression of Ifi204 ( $P<0.05$, Ifi204 LV-EPCs: $21 \pm 4$ vs Ifi204 siRNAEPCs: $11 \pm 2$ ), without reaching a significant difference when compared with nonmodified EPCs (Fig. 5b).

\section{Reperfusion of blood flow in ischemic hindlimb was hindered by transplantation of Ifi204 siRNA-transfected EPCs}

To evaluate the neovascularization in response to Ifi204 modulation in EPCs, we used the hindlimb ischemia model. The laser Doppler blood flow measurement was performed immediately after ligation and 14 days after surgery and cell transplantation (Fig. 6a). The blood flow recovery ratios of ischemia to nonischemia were used as an index of the neovascularization following hindlimb ischemia. Initial reductions in perfusion were similar in all groups (Fig. 6a). Perfusion recovered incrementally over a 2-week period. Transplantation with normal EPCs and
Ifi204 LV-EPCs presented similar blood flow recovery (normal EPCs: $85 \pm 0.05 \%$ vs. Ifi204 LV-EPCs: $93 \pm 0.03 \%$, NS) (Fig. 6a, b). In comparison with this, transplantation with Ifi204 siRNA-transfected EPCs had significantly less blood flow recovery as assessed by laser Doppler $(P<0.05$, Ifi204 siRNA-EPCs: $72 \pm 0.02 \%$ vs normal EPCs: $85 \pm 0.05 \%$; $P$ $<0.01$, Ifi204 siRNA-EPCs: $72 \pm 0.02 \%$ vs Ifi204 LV-EPCs: $93 \pm 0.03 \%$ ), indicating that interference of Ifi204 expression in EPCs may prohibit their vascular reparative ability.

In-vivo vasculogenic activity of EPCs was modulated by the expression of Ifi204

Revascularization of the ischemic hindlimb during the 14 postoperative days was additionally evaluated by immunohistochemistry. The capillaries in the ischemic area are visualized by immunofluorescent staining with an antibody to isolectin B4, which stains endothelial cells, and counted in representative high-power fields (HPF) (Fig. 7a). The mice transplanted with Ifi204 overexpressing EPCs and normal EPCs exhibited sufficient growth of neovessels, while the mice transplanted with Ifi204 siRNA-

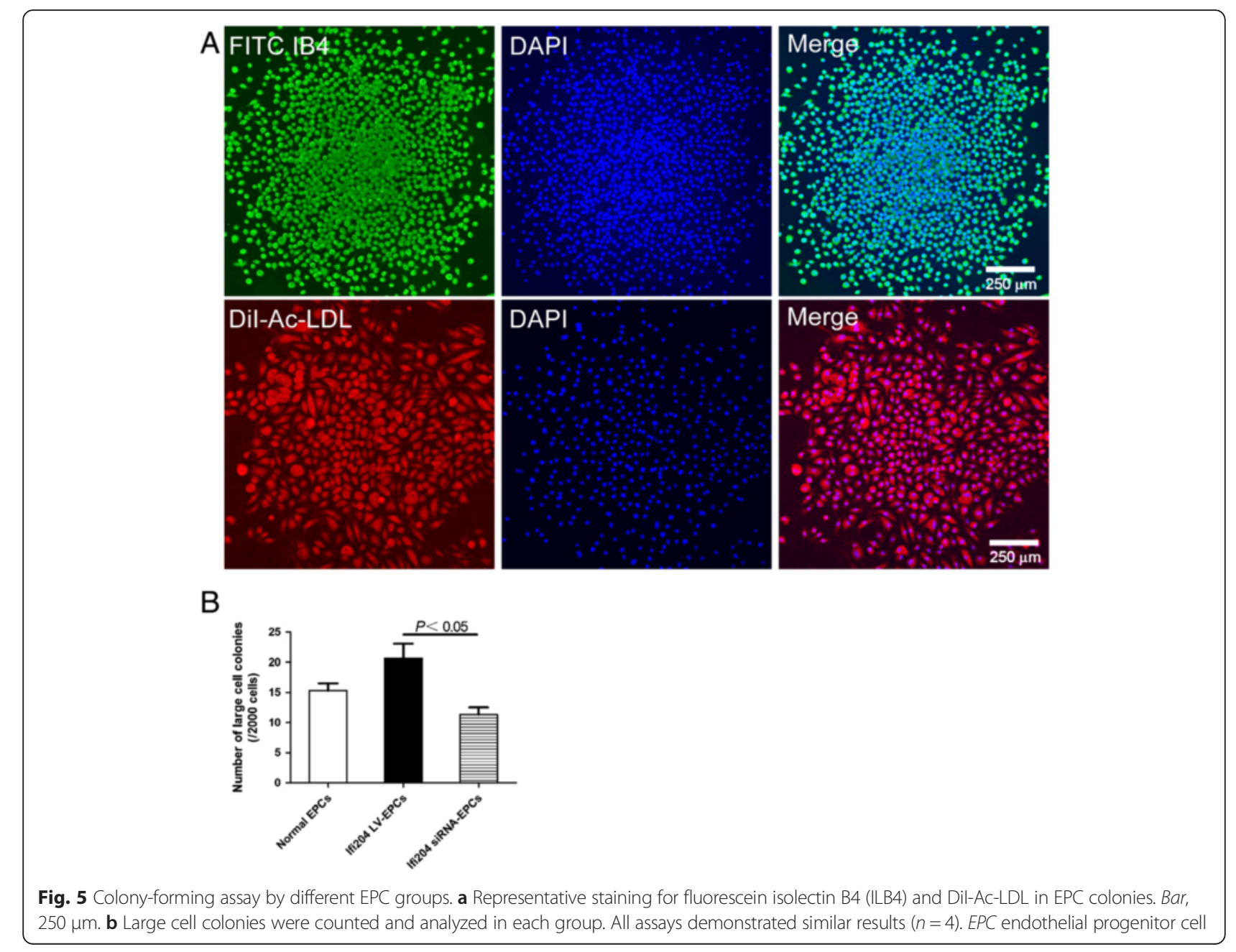



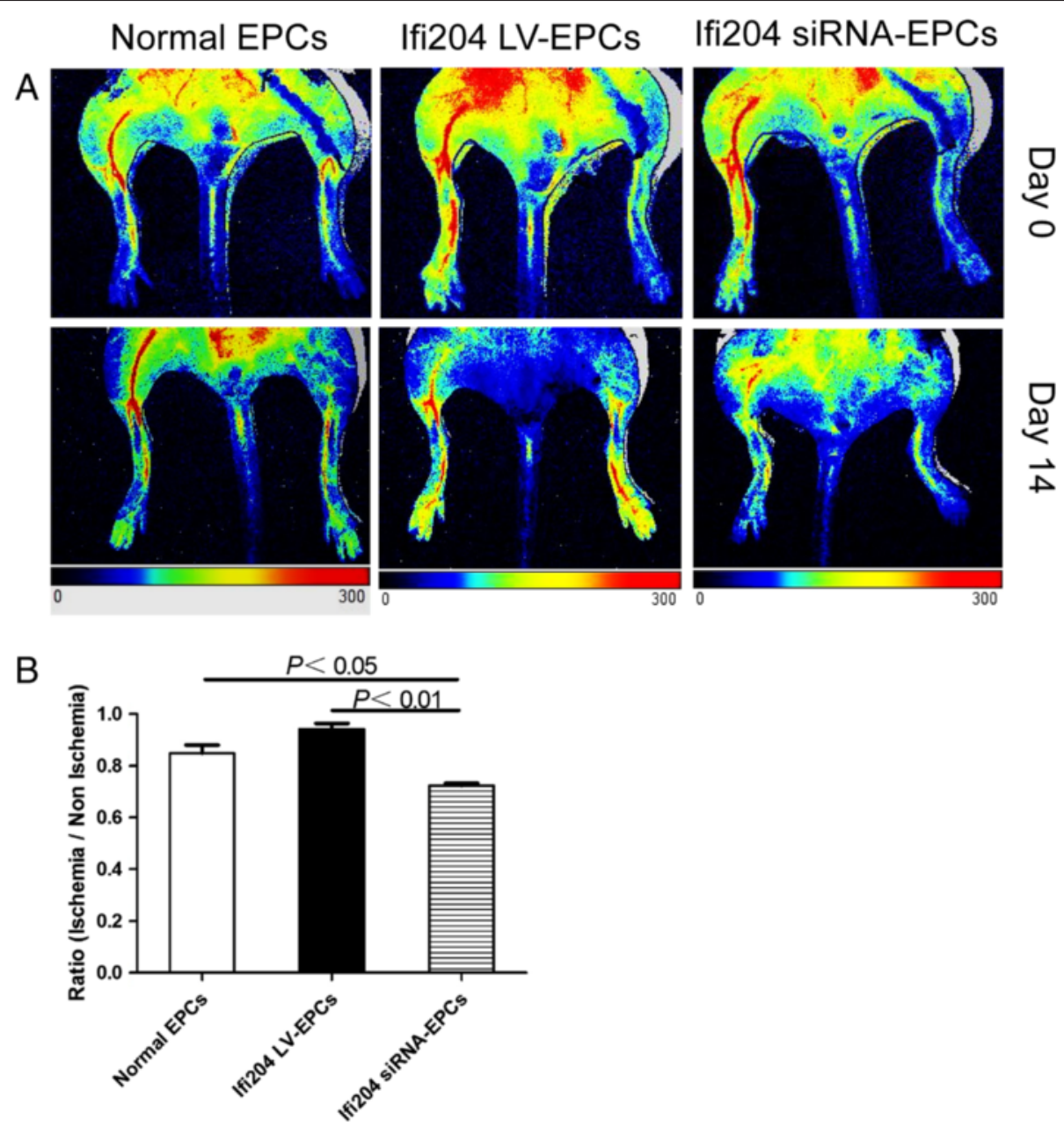

Fig. 6 Blood flow patterns in ischemic hindlimbs. a LDPI was used to analyze blood flow 14 days after ischemia. Top panel: representative features just after surgery. Colors displayed correspond to intervals of perfusion value from 0 (dark) to 300 (red). Red, highest velocity; green, intermediate; blue, low; and dark, lowest velocity. b Blood-flow ratio of ischemic-to-contralateral hindlimb after 14 days $(n=6)$. $P<0.05$, normal EPCs vs Ifi204 siRNAEPCs; $P<0.01$, Ifi204 LV-EPCs vs Ifi204 siRNA EPCs. EPC endothelial progenitor cell (Color figure online)

transfected EPCs exhibited less neoangiogenesis $(P<$ 0.001, Ifi204 siRNA-EPCs: $13 \pm 1.7 / \mathrm{HPF}$ vs normal EPCs: $24 \pm 1.7 / \mathrm{HPF}$ and Ifi204 LV-EPCs: $27 \pm 2.5 / \mathrm{HPF}$ ) (Fig. $7 \mathrm{~b}$ ). A newly-synthesized nanofluorogen, a bolaamphiphile with a tetraphenylethene (TPE) unit attached and two pyridinium salt terminated alkyl groups (TPE-11), was used to track the implanted cells. The fluorescent spots can be clearly seen in the cytoplasmic area of EPCs at the excitation wavelength of $405 \mathrm{~nm}$, and the intensity in the nuclei region is relatively weak (Fig. 8a). In the sections of ischemic hindlimb, we could also detect the cells with TPE-11 labeling (Fig. 8b). EPCs with overexpression of Ifi204 incorporated more into vessel formation, but downexpression of Ifi204 inhibited this stimulatory effect under the control level (Fig. 9a, b). These data indicated that the stimulation of angiogenesis induced by EPCs in ischemic hindlimb was related with the expression level of Ifi204.

\section{Discussion}

Interferon-inducible protein 204 (Ifi204) was shown to be an important mediator for the differentiation of osteoblasts [24-26], skeletal muscle myoblasts [16, 27], and cardiomyocytes [28, 29], previously. Ifi204 has been documented to overcame the inhibition of transcription factors such as Gata4, Nkx2.5, MyoD, and Cdfa1 by sequestering inhibition of differentiation (Id) proteins, thus promoting differentiation of cardiomyocytes and osteoblasts [17]. However, the role of Ifi204 in differentiation of EPCs remains unknown. Very recently, our group reported that Ifi204 is downregulated in the bone marrow cells of diabetes mellitus type 2 mice [18]. Because EPCs in diabetes mellitus have been reported to be impaired in regenerative potency, we thus proposed that Ifi204 could modulate the differentiation and regenerative abilities of EPCs. Our study indicates that 

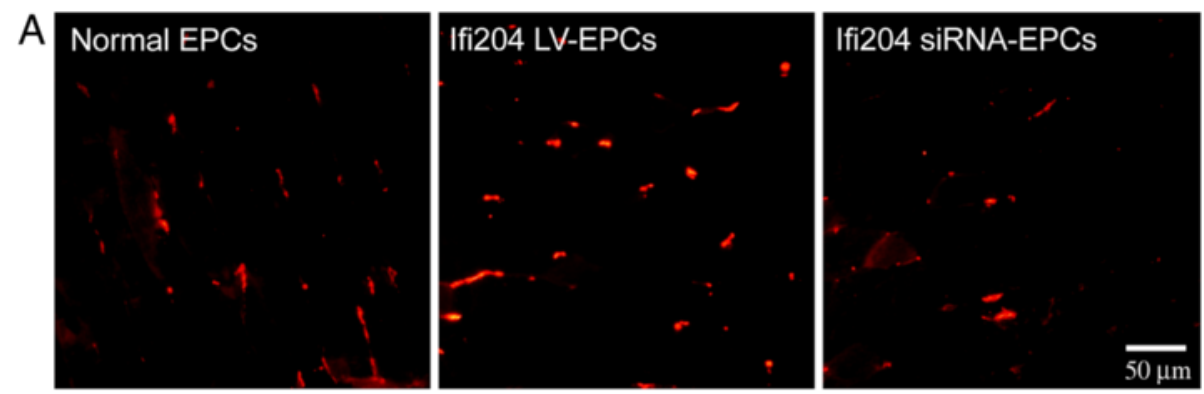

$\mathrm{B}$

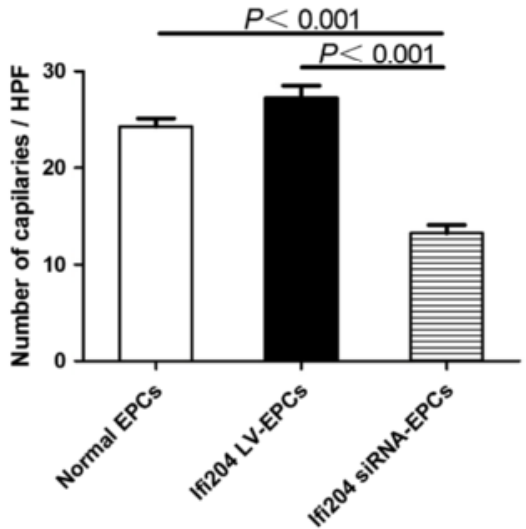

Fig. 7 Histological analysis for capillary density in ischemic hindlimb. Cells were intramuscularly injected immediately after hindlimb ischemia. Muscle samples were harvested following BSL I systemic perfusion 14 days after surgery. a Representative images of vessel morphology from each treatment group. Bar, $50 \mu \mathrm{m}$. b Numbers of capillaries were counted in the ischemic area and averaged in each group $(n=4)$, respectively. EPC endothelial progenitor cell
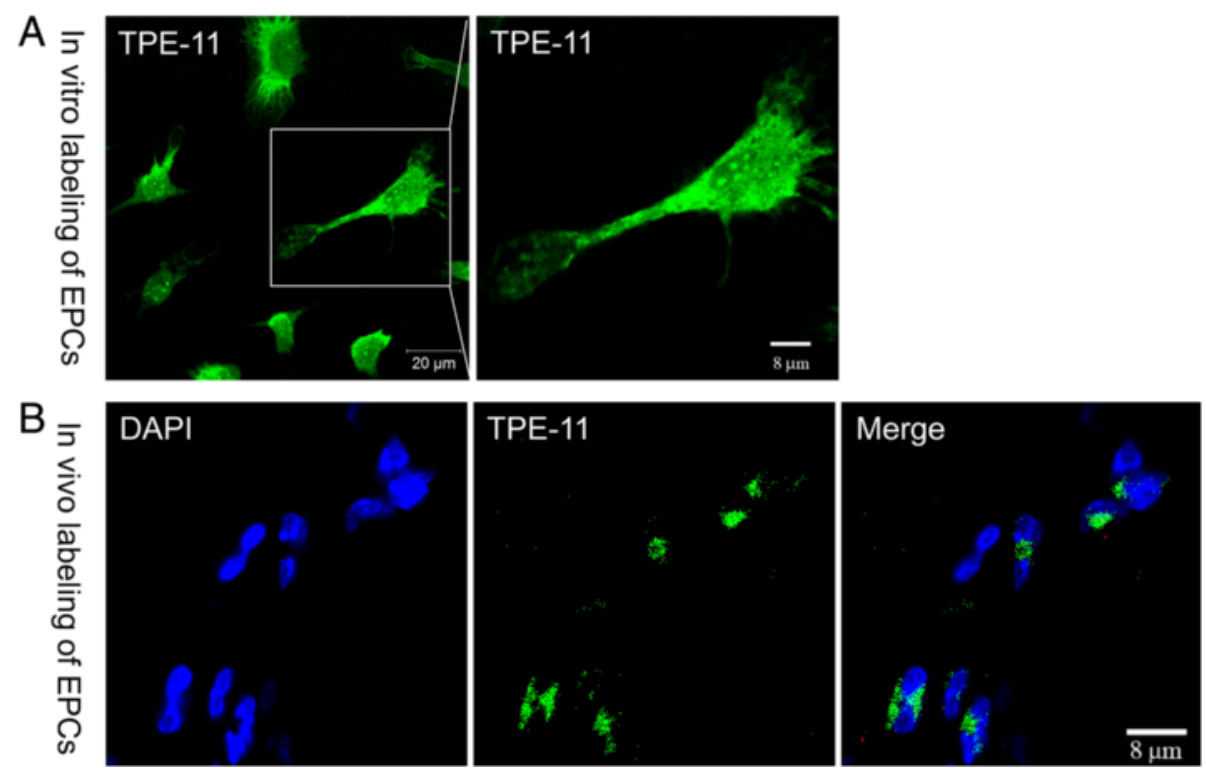

Fig. 8 In-vitro and in-vivo staining of EPCs by the nanofluorogen. a EPCs were incubated with TPE-11 for 12 hours. Fluorescent images of the cells observed by a confocal microscope at the $405 \mathrm{~nm}$ excitations. Bar, $20 \mu \mathrm{m}$ and $8 \mu \mathrm{m}$. b Sections of the ischemic gastrocnemius muscle were observed by a confocal microscope at the $405 \mathrm{~nm}$ excitations. Bar, $8 \mu \mathrm{m}$. EPC endothelial progenitor cell 


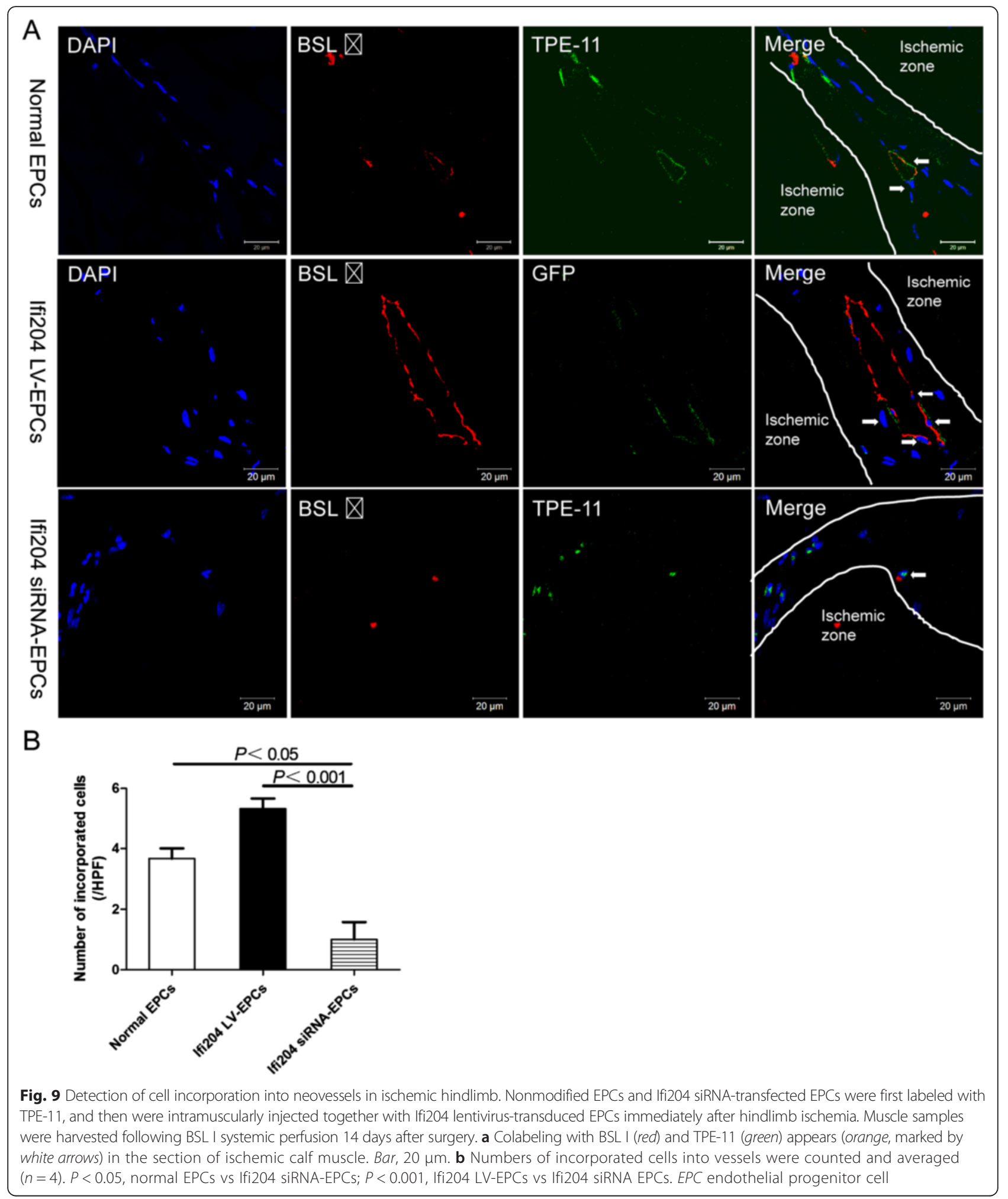

downregulation of Ifi204 in EPCs significantly inhibited their endothelial differentiation and the angiogenic potencies in vitro and in vivo, while upregulation of Ifi204 had the opposite effects. To our knowledge, this is the first study into the regulatory role of Ifi204 on the differentiation and functions of EPCs.

As evidenced by RT-qPCR, immunocytochemistry, and FACS, upregulation of Ifi204 in EPCs promoted 
endothelial differentiation of the cells and vice versa. One of the paracrine factors of VEGF was also secreted more in EPCs infected with Ifi204 lentivirus compared with nonmodified and Ifi204 siRNA transfected cells, whereas cell proliferation was not affected by Ifi204 expression levels (Additional file 1: Figure S1). The mechanisms for how Ifi204 affects the differentiation and paracrine signature of EPCs were unclear and worthy of further investigation. EPCs by themselves do not generate tubes in vitro in Matrigel [30], so researchers utilized EPCs and HUVECs to perform the Matrigel assay [19-21]. Vascular tube formation in Matrigel is thought to recapitulate in-vivo vasculogenesis and angiogenesis [30]. The more angiogenic potencies that EPCs have, the more easily the cells could incorporate into tubular structures of HUVECs. An in-vitro incorporation study also demonstrated that the Ifi204 lentivirus-transduced EPCs could incorporate more into the tubes of HUVECs. To verify the effect of Ifi204, modified EPCs were transplanted into ischemic hindlimb. In mice with hindlimb ischemia, downregulation of Ifi204 in EPCs impaired endothelial differentiation, with a corresponding reduction in neovascularization and hindlimb blood reperfusion by postoperative day 14 . The present study provided evidence that Ifi204 is required for endothelial differentiation of EPCs and the angiogenesis ability of EPCs was modulated by the expression of Ifi204. These findings are consistent with previous reports from other researchers that Ifi204 has participated in the regulation of cell differentiation. Further studies elucidating the downstream signaling molecules of Ifi204 in the modulation of EPC differentiation are needed.

EPCs can form colony-forming units (CFU) in methylcellulose supplied with growth factors. The colony-forming assay determines the stemness of EPCs, and colonies are classified into two types according to their morphology: primitive (small cell) colonies and definitive (large cell) colonies $[19,31]$. The primitive CFU is a predominantly proliferative population of cells and the definitive CFU is a predominantly vasculogenic population with greater adhesion, migration, differentiation, and tabularization potential. EPCs downexpressing Ifi204 formed less large cell colonies than those overexpressing Ifi204, which indicates that Ifi204 inhibition may block the stemness and vasculogenic potency of EPCs and supports all of our findings.

TPE-11 is a nanofluorogen newly synthesized by our group. We have already used it in the labeling of Hela cells, and the biocompatibility of the nanofluorogens formed by TPE-11 was quite good in cells [23]. TPE-11 can be transported and passaged to the progeny cells by microtubules (unpublished observations). We also found that TPE-11 can be retained stably in the embryoid of the embryonic stem cells (unpublished observations: Shixin Zhou (S. Z.), Yijun Xia (Y. X.), Yinan Liu (Y. L.),
Qihua He (Q. H.) and Bo Song (B. S.). 2016.). As far as we are aware, this work represents the first experimental demonstration of in-vivo cell labeling using this emerging luminescent system.

\section{Conclusions}

This study makes two contributions to EPC-mediated therapy of hindlimb ischemia. First, Ifi204 is required for EPC differentiation and neovascularization in vitro and in vivo. Second, EPCs labeled with TPE-11 could be detected in the ischemic hindlimb. The regulatory roles of Ifi204 in EPC differentiation need to be elucidated further and may benefit the therapeutic strategies for ischemic vascular diseases.

\section{Additional file}

Additional file 1: Figure S1. Showing the detection of cell proliferation by CCK-8 assay. A Cells were transfected with Ifi204 siRNA and control siRNA. OD values were read after 2 hours of incubation with CCK-8 solution. B Cells were infected with Ifi204 LV5 and control LV5. OD values were read after 2 hours of incubation with CCK-8 solution. (JPG $41 \mathrm{~kb}$ )

\begin{abstract}
Abbreviations
BSL I, Griffonia (Bandeiraea) Simplicifolia Lectin I; CFU, colony-forming unit; EPC, endothelial progenitor cell; FACS, fluorescence-activated cell sorting; Ifi204 LV-EPCs, EPCs transduced with Ifi204 lentivirus; Ifi204 siRNA-EPCs, EPCs transfected with Ifi204 siRNA; Ifi204, interferon-inducible protein 204; LDPI, laser Doppler perfusion imaging; RT-qPCR, reverse transcription quantitative PCR; TPE, tetraphenylethene; HUVEC, human umbilical vein endothelial cell; TPE11, a bolaamphiphile with a tetraphenylethene unit attached and two pyridinium salt terminated alkyl groups; HPF, high-power field
\end{abstract}

\section{Funding}

This work was supported by the National Natural Science Foundation of China (No. 81400199 and No. 30972696), the Natural Science Foundation of Jiangsu Province (BK20150320), and the Suzhou Municipal Science and Technology Project of China (No. SYS201414).

Availability of data and materials

The datasets supporting the conclusions of this article are included within the article.

\section{Authors' contributions}

$J Y, X Z, Z Z, X L, X W$, and $M C$ were responsible for performance of experiments, data analysis, and manuscript preparation. BS was responsible for nanofluorogen synthesis, cell labeling, and manuscript writing. MI, ZS, and JY were responsible for manuscript writing and revision, and experimental design. All authors read and approved the final manuscript.

\section{Competing interests}

The authors declare that there is no conflict of interests regarding the publication of this article.

\section{Consent for publication}

All authors have contributed to, read, and approved the final manuscript for submission.

\section{Author details}

${ }^{1}$ Institute for Cardiovascular Science \& Department of Cardiovascular Surgery of The First Affiliated Hospital, Soochow University, 188 Shizi Street, Suzhou 215006, China. Department of Cardiovascular Surgery, Affiliated Shanghai 1st People's Hospital, Shanghai Jiaotong University, Shanghai 200080, China. ${ }^{3}$ Suzhou Key Laboratory of Macromolecular Design and Precision Synthesis, Jiangsu Key Laboratory of Advanced Functional Polymer Design and Application, College of Chemistry, Chemical Engineering and Materials Science, Soochow University, Suzhou 215006, China. ${ }^{4}$ Division of Research Animal Laboratory and Translational Medicine, 
Research and Development Center, Osaka Medical College, 2-7 Daigaku-machi, Takatsuki, Osaka 569-8686, Japan. ${ }^{5}$ Institute for Cardiovascular Science, Soochow University, 708 Renmin Road, Suzhou 215006, China.

Received: 12 May 2016 Revised: 9 July 2016

Accepted: 14 July 2016 Published online: 11 August 2016

\section{References}

1. Asahara T, Murohara T, Sullivan A, Silver M, van der Zee R, Li T, et al. Isolation of putative progenitor endothelial cells for angiogenesis. Science. 1997;275:964-7.

2. Asahara T, Masuda H, Takahashi T, Kalka C, Pastore C, Silver M, et al. Bone marrow origin of endothelial progenitor cells responsible for postnatal vasculogenesis in physiological and pathological neovascularization. Circ Res. 1999:85:221-8.

3. Masuda H, Kalka C, Takahashi T, Yoshida M, Wada M, Kobori M, et al. Estrogen-mediated endothelial progenitor cell biology and kinetics for physiological postnatal vasculogenesis. Circ Res. 2007;101:598-606.

4. Rafii S. Circulating endothelial precursors: mystery, reality, and promise. J Clin Invest. 2000;105:17-9.

5. Rafii S, Lyden D. Therapeutic stem and progenitor cell transplantation for organ vascularization and regeneration. Nat Med. 2003:9:702-12.

6. Dai Y, Ashraf M, Zuo S, Uemura R, Dai YS, Wang Y, et al. Mobilized bone marrow progenitor cells serve as donors of cytoprotective genes for cardiac repair. J Mol Cell Cardiol. 2008;44:607-17.

7. Ii M, Nishimura H, Iwakura A, Wecker A, Eaton E, Asahara T, et al. Endothelial progenitor cells are rapidly recruited to myocardium and mediate protective effect of ischemic preconditioning via "imported" nitric oxide synthase activity. Circulation. 2005;111:1114-20.

8. Losordo DW, Dimmeler S. Therapeutic angiogenesis and vasculogenesis for ischemic disease: part II: cell-based therapies. Circulation. 2004;109:2692-7.

9. Murasawa S, Llevadot J, Silver M, Isner JM, Losordo DW, Asahara T. Constitutive human telomerase reverse transcriptase expression enhances regenerative properties of endothelial progenitor cells. Circulation. 2002;106:1133-9.

10. Urbich C, Aicher A, Heeschen C, Dernbach E, Hofmann WK, Zeiher AM, et al. Soluble factors released by endothelial progenitor cells promote migration of endothelial cells and cardiac resident progenitor cells. J Mol Cell Cardiol. 2005;39:733-42.

11. Iwaguro H, Yamaguchi J, Kalka C, Murasawa S, Masuda H, Hayashi S, et al. Endothelial progenitor cell vascular endothelial growth factor gene transfer for vascular regeneration. Circulation. 2002;105:732-8.

12. Jiang M, Wang B, Wang C, He B, Fan H, Guo TB, et al. Angiogenesis by transplantation of HIF-1 alpha modified EPCs into ischemic limbs. J Cell Biochem. 2008;103:321-34.

13. Kong D, Melo LG, Mangi AA, Zhang L, Lopez-Hasaca M, Perrella MA, et al. Enhanced inhibition of neointimal hyperplasia by genetically engineered endothelial progenitor cells. Circulation. 2004;109:1769-75.

14. Kusano KF, Pola R, Murayama T, Curry C, Kawamoto A, Iwakura A, et al. Sonic hedgehog myocardial gene therapy: tissue repair through transient reconstitution of embryonic signaling. Nat Med. 2005;11:1197-204.

15. Asefa B, Klarmann KD, Copeland NG, Gilbert DJ, Jenkins NA, Keller JR. The interferon-inducible p200 family of proteins: a perspective on their roles in cell cycle regulation and differentiation. Blood Cells Mol Dis. 2004;32:155-67.

16. Liu C, Wang H, Zhao Z, Yu S, Lu YB, Meyer J, et al. MyoD-dependent induction during myoblast differentiation of p204, a protein also inducible by interferon. Mol Cell Biol. 2000;20:7024-36.

17. Ding B, Liu CJ, Huang Y, Hickey RP, Yu J, Kong W, et al. p204 is required for the differentiation of P19 murine embryonal carcinoma cells to beating cardiac myocytes: its expression is activated by the cardiac Gata4, Nkx2.5, and Tbx5 proteins. J Biol Chem. 2006;281:14882-92.

18. Yang J, Yan W, Ren C, Li X, Zhang Y, Asahara T, et al. Genome-wide differential gene expression profiling of bone marrow cells in diabetes mellitus type 2 mice. Int J Clin Exp Med. 2016:9:5574-83.

19. Yang J, li M, Kamei N, Alev C, Kwon SM, Kawamoto A, et al. CD34+ cells represent highly functional endothelial progenitor cells in murine bone marrow. PLoS One. 2011;6:e20219.

20. Asai J, Takenaka H, Kusano KF, li M, Luedemann C, Curry C, et al. Topical sonic hedgehog gene therapy accelerates wound healing in diabetes by enhancing endothelial progenitor cell-mediated microvascular remodeling. Circulation. 2006;113:2413-24.
21. Ye Y, Li X, Zhang Y, Shen Z, Yang J. Androgen modulates functions of endothelial progenitor cells through activated Egr1 signaling. Stem Cells Int. 2016;2016:7057894.

22. Kalka C, Masuda H, Takahashi T, Kalka-Moll WM, Silver M, Kearney M, et al. Transplantation of ex vivo expanded endothelial progenitor cells for therapeutic neovascularization. Proc Natl Acad Sci U S A. 2000;97:3422-7.

23. Xia $Y$, Dong $L$, Jin $Y$, Wang $S$, Yan $L$, Yin $S$, et al. Water-soluble nanofluorogens fabricated by selfassemblyof bolaamphiphiles bearing AIE moieties: towards application in cell imaging. J Mater Chem B. 2015;3:491-7.

24. Liu CJ, Chang E, Yu J, Carlson CS, Prazak L, Yu XP, et al. The interferoninducible p204 protein acts as a transcriptional coactivator of Cbfa1 and enhances osteoblast differentiation. J Biol Chem. 2005;280:2788-96.

25. Luan $Y, Y u X P, X u$ K, Ding B, Yu J, Huang Y, et al. The retinoblastoma protein is an essential mediator of osteogenesis that links the p204 protein to the Cbfa1 transcription factor thereby increasing its activity. J Biol Chem. 2007;282:16860-70.

26. Luan Y, Yu XP, Yang N, Frenkel S, Chen L, Liu CJ. p204 protein overcomes the inhibition of core binding factor alpha-1-mediated osteogenic differentiation by Id helix-loop-helix proteins. Mol Biol Cell. 2008;19:2113-26.

27. Liu CJ, Ding B, Wang H, Lengyel P. The MyoD-inducible p204 protein overcomes the inhibition of myoblast differentiation by ld proteins. Mol Cell Biol. 2002:22:2893-905.

28. Lim JY, Kim WH, Kim J, Park SI. Induction of Id2 expression by cardiac transcription factors GATA4 and Nkx2.5. J Cell Biochem. 2008;103:182-94.

29. Moskowitz IP, Kim JB, Moore ML, Wolf CM, Peterson MA, Shendure J, et al. A molecular pathway including Id2, Tbx5, and Nkx2-5 required for cardiac conduction system development. Cell. 2007;129:1365-76.

30. Timmermans F, Plum J, Yoder MC, Ingram DA, Vandekerckhove B, Case J. Endothelial progenitor cells: identity defined? J Cell Mol Med. 2009;13:87-102.

31. Tanaka R, Vaynrub M, Masuda H, Ito R, Kobori M, Miyasaka M, et al. Qualitycontrol culture system restores diabetic endothelial progenitor cell vasculogenesis and accelerates wound closure. Diabetes. 2013;62:3207-17.

\section{Submit your next manuscript to BioMed Central and we will help you at every step:}

- We accept pre-submission inquiries

- Our selector tool helps you to find the most relevant journal

- We provide round the clock customer support

- Convenient online submission

- Thorough peer review

- Inclusion in PubMed and all major indexing services

- Maximum visibility for your research

Submit your manuscript at www.biomedcentral.com/submit 